\title{
Significance of Fingerprints in A Brutal Travel Bag Murder- A Case Report
}

\author{
Mohammad Ahmad Abdullah Ahmad AlSuwaidi' ${ }^{1}$, Abdulrahman Mohammed Obaid Almehiri², \\ Mohammed Thani Rashid Almarri ${ }^{1}$, Jassim Mohammed Abdullah Rashid ${ }^{3}$, Bhoopesh Kumar Sharma ${ }^{4}$ \\ ${ }^{1}$ Lieutenant and Assistant Expert, General Department of Forensic Sciences and Criminology, Dubai Police \\ General Head Quarters, Dubai, United Arab Emirates, ${ }^{3}$ Lieutenant Colonnel and Senior Expert, General \\ Department of Forensic Sciences and Criminology, Dubai Police General Head Quarters, Dubai, United Arab \\ Emirates, ${ }^{4}$ Associate Professor, Department of Forensic Science Amity University Dubai, United Arab Emirates
}

\begin{abstract}
Forensic Science can be defined as the application of science to law. On the crime scene, sometimes the odour of the evidence plays a vital role in the location and identification of the evidences. The present case is one of the best examples of this. Dubai police were informed about a bad smell emanating from an apartment in one locality. After an investigation, the investigators and the forensic expert discovered that the smell was from a decomposing human body. Immediately the fingerprints experts were called to the crime scene to collect, identify, and analyze all the fingerprints encountered. This case reflects the precision, accuracy, and reliability of the fingerprint expert and the technology used by them, including chemical methods like ninhydrin and cyanoacrylate along with the high efficacy of the Automated Fingerprint Identification System. The case was uniquely solved with the aid of fingerprint analysis.
\end{abstract}

Keywords: Crime Scene, Fingerprints, cyanoacrtylate, Ninhydrin, Automated Fingerprint Identification System.

\section{Introduction}

Fingerprint evidence has been acknowledged as the gold standard in forensic science for more than a century ${ }^{1}$. Fingerprints are the patterns created on the fingertips by the raised papillary ridges with rows of pores connected to the sweat glands ${ }^{2}$. A fingerprint is a science of using an individual's physical representative to identify the person. It's unique as no two individuals were found to have the same fingerprint ${ }^{3}$. On the other hand, this case is also a typical case based on the particular type of odour (smell) from the dead body, that had served a significant mean of locating the evidence in the presented case. When odor molecules stick to the cell membrane, odors are sensed - this process is known as adsorption. The objective of this case is to

\section{Corresponding Author:}

\section{Dr. Bhoopesh Kumar Sharma,}

Associate Professor, Forensic Science, Amity

University Dubai, Dubai International Academic City.

Email: bsharma@amityuniversity.ae

Contact No. +971-552154972 show the accuracy, determination and expertise of the experts at the General Department Of Forensic Science and Criminology also, how the investigator can use the primary evidences as basic tools in the criminal investigation and identification.

\section{Background of the Case}

On the 19th of April 2017, Dubai police received a call regarding a fouling stink approaching from an apartment in one of the locality. After investigation, it was discovered that the smell was arousing from a decomposing human body. Further, it was found that a woman had been murdered and the body was hidden inside a travel trolly bag in her apartment (figure 1-2). Immediately the fingerprint experts were called in to collect all essential evidences from the crime scene to be tested for latent fingerprint by appropriate technique. 


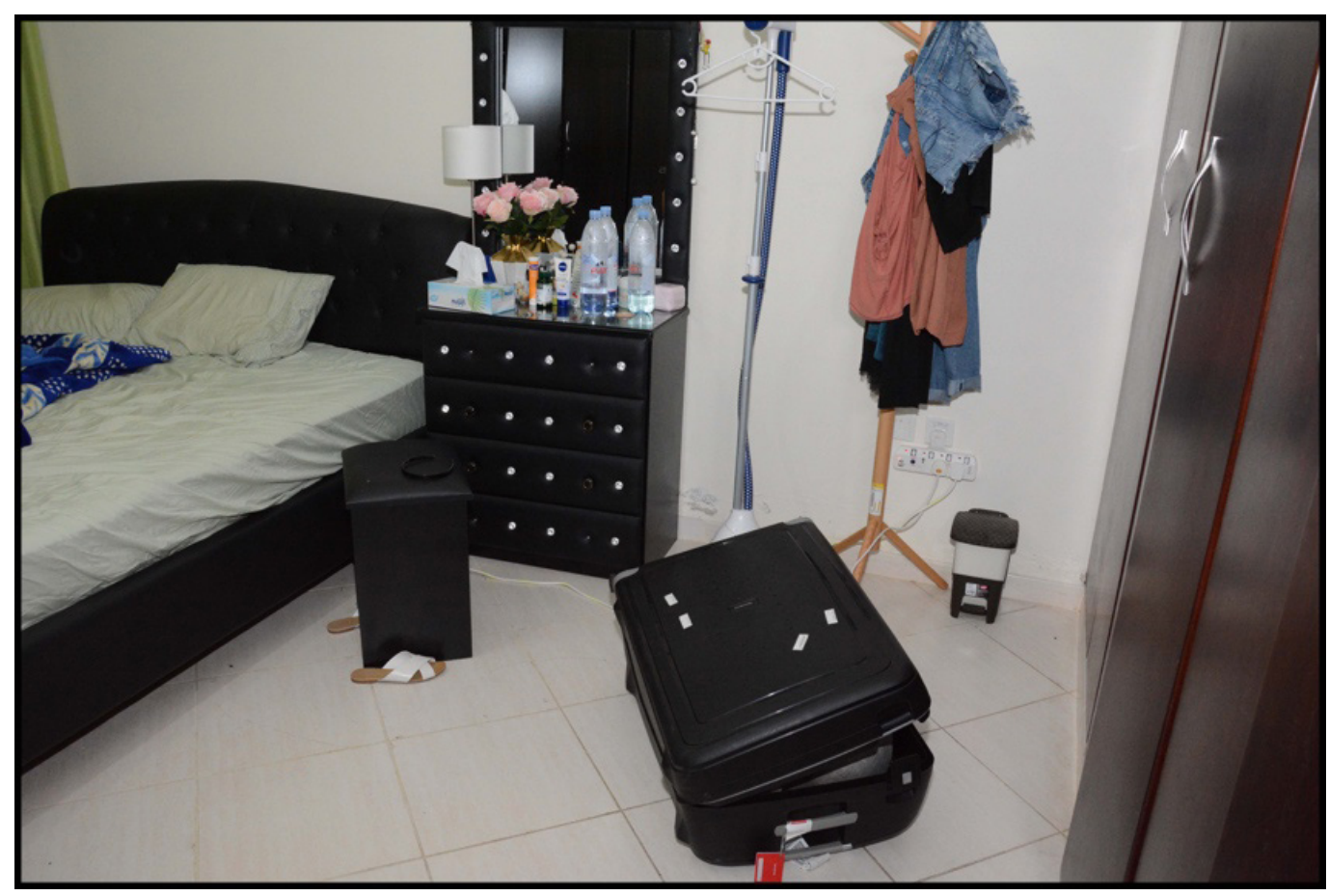

Figure 1: Image of the apartment from where the fouling odour was approaching to the nearby surroundings. The suspected travel bag was lying on the floor.

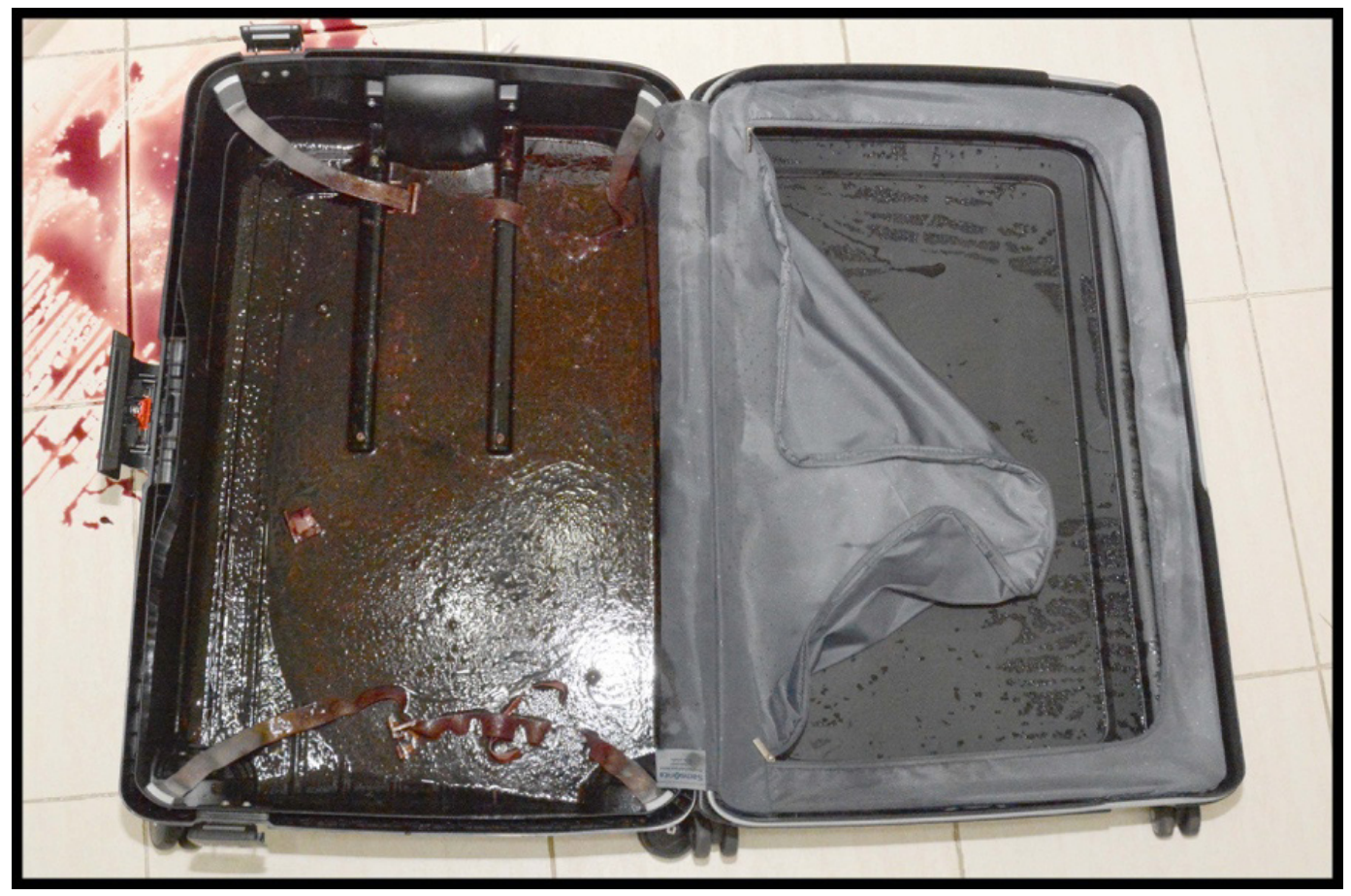

Figure 2: The picture of the blood-stained travel trolly bag, where the dead body of the victim was kept, and fingerprints were collected from the bag and the surrounding areas.

\section{Challenges for the Investigators}

The body becomes flaccid, and blood ceases to circulate after the cessation of heart function. The body undergoes some well-documented modifications, known as the livor, rigor, and algor mortis "classic triad".
Typically, a human body takes between one to three days to start decomposing after death ${ }^{4}$. The rate at which the body decays depends on several factors; how intact the corpse was at the time of death, the temperature and humidity of the environment where the body is kept. If 
the body had skin breakages/injuries at the time of death, the body decomposes faster and smells sooner than the latter ${ }^{5}$. On the other hand, if the body is kept in an environment with high temperature and/or high levels of humidity, it decomposes faster and smells sooner than if it were kept in a cold dry environment.

It is plausible that the body at the apartment had been kept there for at least a day and a maximum period of three days. The apartment is usually regulated for temperature and humidity; thus, the body would have decomposed at the standard rate. The fact that the body was in a travel bag shows that the perpetrator(s) were trying to cover up the incidence until they had exited the apartment and its premises. By the fact that the victim's body was found in her apartment hidden in the bag, it is possible that the murder was committed within this apartment. Dubai police confiscated the bag in order to test for latent fingerprints.

\section{Material and Method}

In an attempt to reveal the fingerprints on the travel bag, the Fingerprint Department encountered two challenges. First, the inside part of the bag was covered by the victim's body fluids. This made it challenging to test and detect fingerprints on the inside, which would shed insight into the identification of the murderer. Cleaning of the bag or any similar attempts would result in loss of this vital evidence for the investigation, and the fingerprint experts had to be careful and methodical in identifying the prints. Secondly, the interior design of the travel bag was curved, making it difficult for experts to capture it with digital cameras.

The Fingerprint Department resorted to utilizing two main techniques; cyanoacrylate fuming, and ardrox. Cyanoacrylate fuming method uses the vapor of superglue to detect latent fingerprints on non-porous surfaces including glass, unfinished wood, plastic, and rubber bands ${ }^{6}$. This technique relies on the deposition of polymerized cyanoacrylate esters on the residues of latent fingerprints. After implementing this technique, the latent fingerprints become visible. This technique was implemented on the inside and outside of the travel bag, given that the latter is made of plastic.

\section{Findings}

Consequently, two fingerprints were detected on the outside and inside of the bag. The prints, however, were not clear enough for the analysis and the decipherment.
This necessitated the use of a second technique; ardrox. Ardrox is a fluorescent liquid dye that stains prints detected using the cyanoacrylate technique. Ardrox works well with Ultra Violet (UV) light, lasers as well as other alternative light sources. When used with cyanoacrylate, ardrox forms a compound that emits secondary radiations under UV light ${ }^{7}$. This compound enables experts to view and photograph fingerprints on a multi-colored and non-porous materials ${ }^{8}$.

After applying ardrox, the fingerprints on the inside and the outside of the travel bag became more explicit, allowing appropriate viewing and photographing. The results of the fingerprint examination revealed a set of prints that were matched those of a suspect.

\section{Conclusion}

The fingerprints still serve as an essential tool for the forensic expert during the investigation of the crime ${ }^{9}$. The most important consideration is the type of print encountered, its condition, nature, and the kind of surface it has been encountered ${ }^{10}$. Many times it is challenging to rely on the fingerprint evidence solely because of lesser ridge details or the inappropriate surface for the development of fingerprints ${ }^{11}$. However, due to the cutting edge technology and trained experts in the field, we can reach conclusive results in many cases as happened in the presented case. The fingerprints of the suspect were found on both the inside and outside of the travel bag, even though they were not identical. Fingerprints are the most valuable sources of evidence for crime owing to their two main features; uniqueness and their never-changing nature ${ }^{12}$. Accordingly, no two individuals have similar prints, and no two fingers of the same person are ever identical. Fingerprints also do not change for the entire of an individual life. The two prints on the travel bag matched those of the suspect and were not identical since they were from different fingers.

The suspect must have murdered the lady in her apartment. This is revealed by the presence of prints on the bag, an indication of involvement in the act. The prints on the inside of the bag indicate that the suspect tried to pack the body in the travel bag. The suspect must have handled the body in the events leading to the demise and hiding of the body in the apartment.

\section{Conflicts of Interest Statement}

The authors whose names are listed immediately below certify that they have NO affiliations with or 
involvement in any organization or entity with any financial interest (such as honoraria; educational grants; participation in speakers' bureaus; membership, employment, consultancies, stock ownership, or other equity interest; and expert testimony or patent-licensing arrangements), or non-financial interest (such as personal or professional relationships, affiliations, knowledge or beliefs) in the subject matter or materials discussed in this manuscript.

\section{Source of Funding: Self}

Acknowledgement: We would like to express our gratitude and appreciation to Senior Expert Dr. Faud Tarbah, head of Research and Development Department for their co-operation and assistance through our work. An, we would like to thank the General Department Of Forensic Science And Criminology to conduct this Case Study.

Ethical Clearance: Ethical clearance has been obtained from Dubai Police HQ's and all the authors.

\section{References}

1. FAULDS H. On the Skin-Furrows of the Hand. Nature. 1880;22(574):605-605.

2. Wertheim K, Maceo A. The critical stage of friction ridge and pattern formation. Journal for Identification. 2002;52:35-85.

3. Ashbaugh D. Quantitative-qualitative friction ridge analysis. Boca Raton, Fla.: CRC Press; 1999.

4. O'Connor T, Boddington A, Garland A, Janaway R. Death, Decay and Reconstruction. Approaches to Archaeology and Forensic Science. American
Journal of Archaeology. 1989;93(3):462.

5. Rowe W. Review of:Advances in Forensic Taphonomy: Method, Theory, and Archaeological Perspectives. Journal of Forensic Sciences. 2002;47(6):15590J.

6. Soweon Yoon, Jianjiang Feng, Jain A. Altered Fingerprints: Analysis and Detection. IEEE Transactions on Pattern Analysis and Machine Intelligence. 2012;34(3):451-464.

7. Ji L, Yi Z. Fingerprint orientation field estimation using ridge projection. Pattern Recognition. 2008;41(5):1491-1503.

8. Bumbrah G. Cyanoacrylate fuming method for detection of latent fingermarks: a review. Egyptian Journal of Forensic Sciences. 2017;7(1).

9. Kiran A P. Study on Efficiency of Alternate Light Source for Detection of Latent Fingerprints. Journal of Forensic Sciences \& Criminal Investigation. 2018;9(5).

10. Lymperopoulou K, Nikitakis A. Fun with Fingerprints: Cyanoacrylate Fuming. Journal of Chemical Education. 2008;85(6):816A.

11. Friesen J. Activities Designed for Fingerprint Dusting and the Chemical Revelation of Latent Fingerprints. Journal of Chemical Education. 2014;92(3):505-508.

12. Almog J, Gabay A. Chemical Reagents for the Development of Latent Fingerprints. III: Visualization of Latent Fingerprints by Fluorescent Reagents in Vapor Phase. Journal of Forensic Sciences. 1980;25(2):12146J. 